\title{
Effects of Sleep, Physical Activity, and Shift Work on Daily Mood: a Prospective Mobile Monitoring Study of Medical Interns
}

\author{
David A. Kalmbach, PhD' , Yu Fang, MSE' ${ }^{2}$ J. Todd Arnedt, PhD' , Amy L. Cochran, PhD', \\ Patricia J. Deldin, PhD ${ }^{7}$, Adam I. Kaplin, MD PhD' , and Srijan Sen, MD PhD ${ }^{1,2}$
}

'Department of Psychiatry, University of Michigan Medical School, Ann Arbor, MI, USA; ${ }^{2}$ Molecular and Behavioral Neuroscience Institute, University of Michigan, Ann Arbor, MI, USA; ${ }^{3}$ Department of Mathematics, University of Michigan, Ann Arbor, MI, USA; ${ }^{4}$ Departments of Psychiatry and Neurology, Johns Hopkins University School of Medicine, Baltimore, MD, USA.

BACKGROUND: Although short sleep, shift work, and physical inactivity are endemic to residency, a lack of objective, real-time information has limited our understanding of how these problems impact physician mental health. OBJECTIVE: To understand how the residency experience affects sleep, physical activity, and mood, and to understand the directional relationships among these variables. DESIGN: A prospective longitudinal study.

SUBJECTS: Thirty-three first-year residents (interns) provided data from 2 months pre-internship through the first 6 months of internship.

MAIN MEASURES: Objective real-time assessment of daily sleep and physical activity was assessed through accelerometry-based wearable devices. Mood scaled from 1 to 10 was recorded daily using SMS technology. Average compliance rates prior to internship for mood, sleep, and physical activity were $77.4,80.2$, and $93.7 \%$, and were 78.8, 53.0, and 79.9\% during internship.

KEY RESULTS: After beginning residency, interns lost an average of $2 \mathrm{~h}$ and $48 \mathrm{~min}$ of sleep per week $(t=-3.04$, $p<.01)$. Mood and physical activity decreased by $7.5 \%$ $(t=-3.67, p<.01)$ and $11.5 \%(t=-3.15, p<.01)$, respectively. A bidirectional relationship emerged between sleep and mood during internship wherein short sleep augured worse mood the next day $(b=.12, p<.001)$, which, in turn, presaged shorter sleep the next night $(b=.06, p=.03)$. Importantly, the effect of short sleep on mood was twice as large as mood's effect on sleep. Lastly, substantial shifts in sleep timing during internship (sleeping $\geq 3 \mathrm{~h}$ earlier or later than pre-internship patterns) led to shorter sleep (earlier: $b=-.36, p<.01$; later: $b=-1.75, p<.001)$ and poorer mood (earlier: $b=-.41, p<.001$; later: $b=-.41, p<.001$ ). CONCLUSIONS: Shift work, short sleep, and physical inactivity confer a challenging environment for physician mental health. Efforts to increase sleep opportunity through designing shift schedules to allow for adequate opportunity to resynchronize the circadian system and improving exercise compatibility of the work environment may improve mood in this depression-vulnerable population.

KEY WORDS: medical education; sleep disorders; medical student and residency education.

Yu Fang is a co-lead

Received August 29, 2017

Revised January 3, 2018

Accepted February 9, 2018

Published online March 14, 2018
J Gen Intern Med 33(6):914-20

DOI: $10.1007 / \mathrm{s} 11606-018-4373-2$

(c) Society of General Internal Medicine 2018

\section{INTRODUCTION}

Sleep problems and physical inactivity are important precipitating factors for major depression. ${ }^{1,2}$ Unfortunately, sleep loss and inactivity are common during physician training and, in particular, during the first year of medical residency. ${ }^{3-6}$ Heavy workloads and circadian misaligned shift schedules contribute to high rates of sleep deprivation during internship., $7-10$ Along the same lines, rotating shift work and time constraints represent major barriers to physical activity and exercise in medical residents ${ }^{11}$ with residents substantially less active than both medical students and attending physicians. ${ }^{6,11,12}$

Prior research on both sleep and activity in residents and their relationship with mood has been limited by reliance on subjective retrospective estimates of average sleep duration and activity over periods of weeks or months. Emerging evidence supports the use of objective, real-time data collection to provide critical insights into physician behaviors and effects of workplace environment. ${ }^{13}$ Objective, real-time assessment of sleep and physical activity would advance our understanding of the longitudinal and directional relationships among the duration and timing of sleep, physical activity, and mood changes during internship. Further, these advances hold the potential to inform the nature and timing of interventions needed to reduce the detrimental impacts of sedentariness, sleep deprivation, and misalignment between habitual and shift work-enforced sleep schedules.

The present study investigated changes in mood, sleep, and physical activity from pre-internship levels across the first 6 months of the intern year. We used a daily diary approach to characterize the manner in which accelerometry-based daily physical activity (measured in steps) and nightly sleep duration were associated with daily mood ratings. We hypothesized that shorter sleep duration and less physical activity would lead to future negative mood. We also hypothesized that negative mood and less physical activity would decrease sleep duration the following night. Lastly, we tested for changes in timing of sleep and wake after transitioning to internship, 
and whether misalignment between pre-internship and internship sleep-wake timing was associated with sleep duration or mood. We hypothesized that greater misalignment from baseline sleep patterns would correspond to shorter sleep and poorer mood during internship.

\section{METHODS}

The present study is a prospective web-based survey study, and this report adheres to the Strengthening The Reporting of OBservational studies in Epidemiology (STROBE) guidelines for the reporting of observational studies. This study was approved by the University of Michigan IRB and all subjects provided informed consent after receiving complete description of the study.

\section{Participants}

The Intern Health Study is a multi-site prospective cohort study that follows training physicians through internship (for details, see Guille $(2015)^{14}$ and Sen $\left.(2010)^{3}\right)$. Fifty-three graduates of the University of Michigan Medical School remaining at Michigan for residency in the 2014-2015 and 2015-2016 intern cohorts were enrolled in the Intern Health Study. All 53 of these interns were invited to participate in a biomarker protocol involving daily mood reports and wearing biotracking devices to measure sleep parameters and physical activity. Forty of the invited interns (75\% enrollment) volunteered for this protocol, of whom 33 interns adhered to the study protocol and provided sufficient data for analysis, resulting in 893 daily observations prior to internship, then 1964 daily observations during the first half of intern year (after data cleaning, and listwise deletion and lagging predictors in the mixed models described below).

\section{Procedure and Measures}

Baseline online survey data assessing basic demographic information, including age and sex, were collected at $1-2$ months prior to internship. Subjects reported average weekly work hours at 3 and 6 months of intern year.

Data collection for sleep duration, physical activity, and daily mood ratings began at 2 months before internship, then continued for 6 months into the intern year. Nightly sleep duration was measured using wrist-based accelerometry Fitbit monitoring (Fitbit Inc., San Francisco, CA; the Fitbit One ${ }^{\circledR}$ used in the 2014-2015 cohort and the Fitbit Charge ${ }^{\mathrm{TM}}$ used in the 2015-2016 cohort), which have been evaluated for research purposes and shown to perform as well as validated actigraphic sleep monitors. ${ }^{15,16}$ Sleep episodes with a wake time between 00:00 and 23:59 on 1 day were sleep between prior day and that day, and in fewer cases, could also be daytime nap on that day, and they were defined as the sleep episodes of that day. For days during which more than one sleep episodes were recorded, the longest episode was operationalized as the main sleep episode. In estimating sleep duration, the total sleep time for all sleep episodes on 1 day was summed to capture both the main sleep episode and naps. Fitbit devices were also used to estimate total steps (reported in the unit of $1000 \mathrm{~s}$ ) as an indicator of daily physical activity. ${ }^{17}$ Mood 247.com (Remedy Health Media LLC, New York, NY), a short-message service-based mood tracking system that has shown promise in patient mood charting, ${ }^{18}$ was used to collect mood ratings. Interns were sent automated SMS message at 20:00 daily with the following prompt: 'On a scale of 1 (low) to 10 (high), what was your average mood today?'

Bedtime, wake time, and midsleep (i.e., the halfway point between bedtime and wake time ${ }^{19}$ ) were extracted from the main sleep episode each day. To capture nightly deviations in internship sleep timing from baseline routine, midsleep shifts were operationally defined as the difference in midsleep on a given night during internship and their own personal preinternship baseline midsleep median (median was used instead of mean due to the skewed distribution of midsleep times). Midsleep shifts were defined as delayed when an intern's midsleep timing was later than their own baseline midsleep average, and advanced when an intern's midsleep timing was earlier than baseline midsleep. Nights during which midsleep differed from baseline by $3 \mathrm{~h}$ or more were interpreted as shift work-related sleep changes ( $\geq 3$-h cut-point based on $1 \mathrm{SD}$ in internship midsleep in the present study).

\section{Statistical Analysis}

All statistical analyses were conducted using R (The R Foundation, Vienna, AUT) ${ }^{20}$ Using within-subjects paired $t$ tests, we first tested for changes in average daily mood, sleep duration and timing, and physical activity between preinternship and intern year.

The primary outcomes of interest consisted of repeated daily mood ratings and nightly estimated sleep duration. To account for the time-nested structure of the data, substantive hypotheses were tested using linear mixed modeling, which allows for the simultaneous testing of between- and withinsubjects effects and is robust to missing data common in repeated measures studies. ${ }^{21,} 22$ Between-subjects factors (i.e., level-2 predictors) included age, sex, and internship work hours (shown to associate with mood, sleep, and physical activity in prior reports ${ }^{3,10,23}$ ), as well as pre-internship mean values for daily mood, physical activity (in steps), and sleep duration. Within-subjects factors (i.e., level-1 predictors) centered on daily internship observations of mood, physical activity, and sleep durations. We first predicted daily mood ratings as estimated by the above pre- and within-internship factors. To test directionality between nightly sleep and daily mood, we lagged the predictor level-1 sleep duration for 1 day (i.e., prior day's sleep duration) in estimating mood ratings and controlled for the prior day's mood as a potential confound. Second, we similarly predicted nightly sleep duration as 
estimated by the above factors, with level-1 daily mood as a lagged predictor to the opposite direction, while controlling for the previous night's sleep.

\section{RESULTS}

Thirty-three of 40 subjects provided sufficient data to be included in the analysis ( 16 women; aged $27.3 \pm 2.6$ years; see Table 1 for full sample characteristics). Of these 33 individuals, one subject did not report gender, but ascertained the subject's biological sex from genetic data (please see Sen et al. $(2010)^{3}$ for DNA collection methods in the Intern Health Study). In addition, three subjects' age was estimated via multivariate imputation. The average compliance rates prior to internship for mood, sleep, and physical activity were 77.4, 80.2 , and $93.7 \%$, while compliance rates were $78.8,53.0$, and $79.9 \%$ during internship.

We first evaluated changes in mood, sleep, and physical activity from baseline to internship (see Table 2 for full results). Within-subjects $t$ tests revealed that daily mood ratings decreased by $7.5 \%(p<.01)$ and physical activity decreased by $11.5 \%(p<.01)$ after transitioning to internship. Interns lost an average of $2 \mathrm{~h}$ and 48 min of sleep per week $(p<.01)$ compared to pre-internship means. Women slept an average of 31 min longer per night than men before internship $(t=2.544$, $p<0.05, d=0.88)$ and 37 min longer per night than men during internship $(t=2.541, p<0.05, d=0.88)$.

\section{Daily Mood, Sleep, and Activity}

We first estimated daily mood based on sleep duration the prior night and physical activity from earlier in the day, while adjusting for average weekly work hours and other relevant demographic and internship factors (Table 3 ). Interns reported better mood after obtaining longer sleep $(b=.12, p<.001$, Fig. 1(a)) and on days, they were more physically active $(b=.04, p<.01$, Fig. 1(b)).

Next, we estimated sleep duration based on mood and physical activity from earlier in the day, while adjusting for the same

Table 1 Sample characteristics $(N=33)$

\begin{tabular}{ll}
\hline \hline Age (years) & $\mathrm{M} \pm \mathrm{SD}, 27.33 \pm 2.58$ \\
Sex (female), $n ; \%$ & $16 ; 48.48$ \\
Race, $n ; \%$ & \\
White & $25 ; 75.76$ \\
Asian or Pacific Islander & $5 ; 15.15$ \\
Multi-racial & $3 ; 9.09$ \\
Specialty, $n ; \%$ & \\
Internal medicine & $4 ; 12.12$ \\
Surgery & $4 ; 12.12$ \\
Obstetrics and gynecology & $3 ; 9.09$ \\
Pediatrics & $4 ; 12.12$ \\
Psychiatry & $3 ; 9.09$ \\
Neurology & $2 ; 6.06$ \\
Emergency medicine & $3 ; 9.09$ \\
Family practice & $1 ; 3.03$ \\
"Other" & $6 ; 18.18$ \\
Transitional & $3 ; 9.09$ \\
\hline
\end{tabular}

$M$ mean, SD standard deviation control factors (Table 4). Results showed that better mood during the day predicted longer sleep duration later that night $(b=.06, p=.03$, Fig. 1(a)). Notably, the impact of sleep on subsequent mood $(b=.12, p<.001$ in the previous model above) was substantially larger than the influence of mood on subsequent sleep ( $b=.06, p=.03$, the present model) in this sample. Contrary to our hypothesis, interns did not sleep longer after being more physically active during the day $(p=.49)$.

\section{Shift Work and Changes in Sleep-Wake Timing}

Next, we evaluated changes in sleep timing during internship (see Table 2 and Fig. 2). On average, midsleep advanced significantly from pre-internship to internship $(\Delta$ Midsleep $=$ $1 \mathrm{~h} 2$ min earlier, $p=.01$ ), reflecting earlier timing of sleep periods during internship. Indeed, $71.7 \%$ of internship midsleep observations (1408 of 1964 observations from 33 subjects) were earlier than average pre-internship patterns. This advanced shift in sleep timing was driven by changes in wake time, with the median wake time occurring $1 \mathrm{~h} 30 \mathrm{~min}$ earlier during internship compared to baseline $(p<.001)$. Conversely, no significant change in median bedtime was observed $(\Delta$ Bedtime $=39$ min earlier, $p=.12)$.

To assess the impact of shift work on sleep duration and mood, we used mixed linear model to evaluate whether changes in sleep-wake timing from baseline to internship that are $\geq$ $3 \mathrm{~h}$ (i.e., $1 \mathrm{SD}$ in intern midsleep as reported in Table 2) were associated with sleep duration and next-day mood. All models controlled for age, sex, and prior night's sleep duration. Results revealed that interns slept shorter on nights when their midsleep was three or more hours earlier $(n=32$, obs $=1408$, no. of earlier shifts $=200, b=-.36, p<.01)$ or later $(n=32$, obs $=552$, no. of later shifts $=199, b=-1.75, p<.001)$ than their baseline sleep patterns. Importantly, advanced shifts led to greater sleep deprivation such that each hour by which midsleep advanced, interns lost an average of $21 \mathrm{~min}$ of sleep $(n=32$, obs $=1229, b=-.35, p<.001)$, compared to $17 \mathrm{~min}$ for each hour of delayed midsleep $(n=28$, obs $=482, b=$ $-.28, p<.001)$. Further, interns reported worse mood on days following a night of misaligned sleep of three or more hours (advanced: $b=-.41, p<.001$; delayed: $b=-.41, p<.001$ ), even after adjusting for the same night's sleep duration. Though both earlier and later sleep schedules presaged negative mood outcomes, results showed that each 1-h advance in midsleep corresponded to a $.18 \mathrm{SD}(-.22 / 1.25, b=-.22$, $p<.001)$ decrease in mood the following day, compared to just a $.03 \mathrm{SD}(-.04 / 1.25, b=-.04, p<.01)$ mood decrease for each hour of delayed shifts.

\section{DISCUSSION}

After transitioning to internship, physicians reported worse mood, shorter nightly sleep, and less physical activity. During internship, day-to-day changes in mood corresponded to fluctuations in physical activity and nightly sleep duration such 
Table 2 Changes in mood, sleep, and physical activity after beginning internship $(N=33)$

\begin{tabular}{|c|c|c|c|}
\hline & Pre-internship & Internship & $t, p$ value, Cohen's $d$ \\
\hline Daily mood (GM \pm GSD) & $7.17 \pm 0.96$ & $6.63 \pm 1.25$ & $t=-3.67, p<.01, d=-.64$ \\
\hline Sleep duration $(\mathrm{GM} \pm \mathrm{GSD})$ & $7 \mathrm{~h} 6 \mathrm{~min} \pm 38 \mathrm{~min}$ & $6 \mathrm{~h} 42 \min \pm 45 \min$ & $t=-3.04, p<.01, d=-.53$ \\
\hline Steps $\times 1000(\mathrm{GM} \pm \mathrm{GSD})$ & $10.15 \pm 2.72$ & $8.98 \pm 2.87$ & $t=-3.15, p<.01, d=-.55$ \\
\hline Bedtime (GMD \pm GSD) & $00: 22 \pm 1.65 \mathrm{~h}$ & $23: 43 \pm 3.14 \mathrm{~h}$ & $t=-1.60, p=.12, d=-.28$ \\
\hline Wake time $(\mathrm{GMD} \pm \mathrm{GSD})$ & $08: 02 \pm 1.64 \mathrm{~h}$ & $06: 32 \pm 2.98 \mathrm{~h}$ & $t=-3.69, p<.001, d=-.64$ \\
\hline Midsleep $(\mathrm{GMD} \pm \mathrm{GSD})$ & $04: 12 \pm 1.46 \mathrm{~h}$ & $03: 10 \pm 2.95 \mathrm{~h}$ & $t=-2.61, p=.01, d=-.45$ \\
\hline
\end{tabular}

$N$, number of subjects; $t$, $t$ statistic for within-subjects $t$ test; $p$ value, statistical significance; Cohen's $d$, effect size; GM, grand mean, i.e., the mean of each subject's mean value on that parameter; GMD, grand median, i.e., the mean of each subject's median value on that parameter, grand median time is presented in 24-h clock in hh:mm format; GSD, grand standard deviation, i.e., the average of each subject's standard deviation on that parameter; $h$, hours; m, minutes

that mood was highest when interns were physically active and well rested. Internship work schedules impacted timing of sleep and wake such that interns woke up, on average, substantially earlier during internship, which presaged reduced sleep duration and worsened mood. Though past studies have explored the consequences of problematic sleep, physical inactivity, and mood in this population, this investigation is the first to measure the association between changes in objectively measured sleep timing and duration and physical activity on daily mood during internship. Critically, our findings add to prior studies $3,4,7,10,24$ suggesting that long work hours, insufficient sleep, and circadian challenging shift schedules may create an insalubrious environment for intern mental health.

Our data confirm and extend to training physicians the bidirectional relationship between daily mood and sleep duration observed in prior subjective studies. We found that when interns obtained shorter sleep, they reported poorer mood the following day, which, in turn, presaged shorter sleep the next night. These results lend objective support to research linking

Table 3 Predictors of daily mood during internship

\begin{tabular}{|c|c|c|c|}
\hline & \multicolumn{3}{|c|}{ Daily mood $(\operatorname{mood} 24 / 7)$} \\
\hline & $b$ & SE & $p$ value \\
\hline Intercept & 0.98 & 2.08 & .64 \\
\hline \multicolumn{4}{|l|}{ Between-subjects effects $(N=33)$} \\
\hline Age & -0.00 & 0.04 & .97 \\
\hline Sex (female) & -0.00 & 0.23 & .99 \\
\hline Mood, pre-internship & 0.71 & 0.12 & $<.001$ \\
\hline Sleep duration, pre-internship & -0.24 & 0.19 & .21 \\
\hline Steps $\times 1000$, pre-internship & -0.09 & 0.04 & .07 \\
\hline Internship work hours & -0.00 & 0.01 & 68 \\
\hline \multicolumn{4}{|c|}{ Within-subjects effects $($ obs $=1964)$} \\
\hline Sleep duration, prior night & 0.12 & 0.02 & $<.001$ \\
\hline Steps $\times 1000$, same day & 0.04 & 0.01 & $<.001$ \\
\hline Mood, previous day & 0.34 & 0.02 & $<.001$ \\
\hline Day in internship (linear)* & -0.10 & 0.03 & $<.01$ \\
\hline \multicolumn{4}{|l|}{ Model fitting } \\
\hline $\mathrm{ICC}$ & 0.16 & & \\
\hline AIC & 6457.53 & & \\
\hline
\end{tabular}

* 'Day in internship' variable was coded as $0,1,2 \ldots n$ with the internship start date $=0$, then converted to standardized $z$-scores to avoid extreme difference in scaling with other variables

$N$, number of subjects; obs, observations, which are smaller here than the total recorded due to lagging of predictor variables and listwise deletion on days during which data were missing; $b$, unstandardized coefficient; ICC, intraclass correlation coefficient; AIC, Akaike information criterion insufficient sleep and negative mood in medical residents, ${ }^{10,24}$ as well as to prior demonstration of reciprocity between daily mood and self-reported nightly sleep in non-resident populations. ${ }^{25,}{ }^{26}$ It is notable that the influence of sleep on mood the next day in our study was substantially larger than the effect of daily mood on sleep the subsequent night. Given that interns experience significant sleep challenges and truncation due to long work hours and circadian misaligned shift schedules, 3,4 , $7,10,23$ these findings highlight the high risk for sleep-related mood perturbations in this population.

Indeed, our data offer critical insights into the effects of internship-related changes in sleep-wake timing and shift work on sleep duration and mood. After transitioning to internship, physicians woke up an average of $1 \mathrm{~h}$ and $30 \mathrm{~min}$ earlier than baseline. However, these earlier wake times during internship were not accompanied by corresponding changes in bedtimes. This finding suggests that insufficient sleep in this

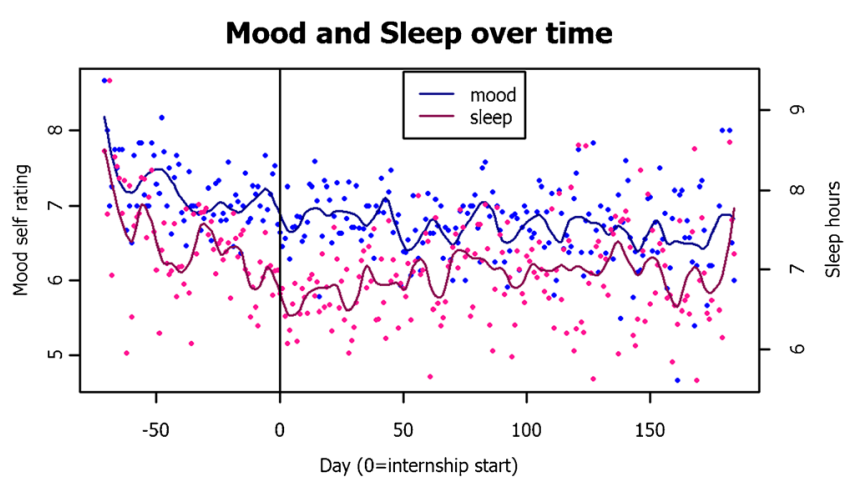

Mood and Physical Activity over time

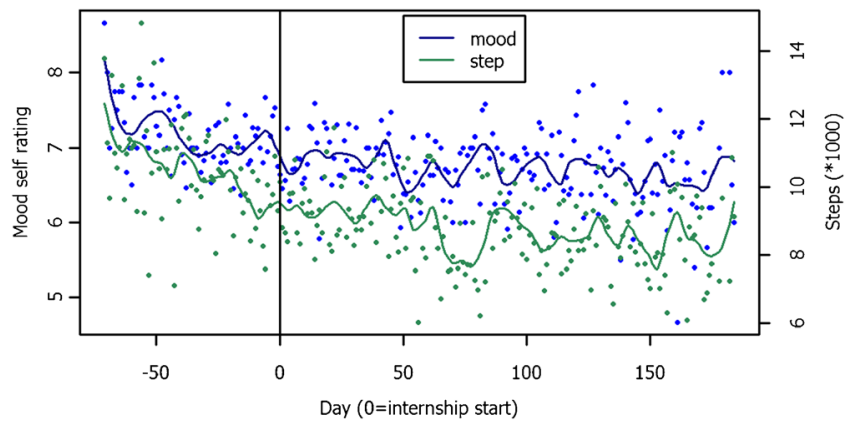

Figure 1 The relationship between mood and sleep, and mood and physical activity through internship. 
Table 4 Predictorsf of nightly sleep duration during internship

\begin{tabular}{|c|c|c|c|}
\hline & \multicolumn{3}{|c|}{ Nightly sleep duration (h) } \\
\hline & $b$ & SE & $p$ value \\
\hline \multirow{2}{*}{\multicolumn{4}{|c|}{ Between-subjects effects $(N=33)$}} \\
\hline & & & \\
\hline Age & -0.03 & 0.04 & 0.44 \\
\hline Sex (female) & 0.26 & 0.23 & 0.28 \\
\hline Mood, pre-internship & -0.24 & 0.13 & 0.07 \\
\hline Sleep duration, pre-internship & 0.49 & 0.20 & 0.02 \\
\hline Steps $\times 1000$, pre-internship & 0.02 & 0.05 & 0.64 \\
\hline Internship work hours & -0.01 & 0.01 & 0.10 \\
\hline \multicolumn{4}{|c|}{ Within-subjects effects (obs $=1964)$} \\
\hline Mood, earlier that day & 0.06 & 0.03 & 0.03 \\
\hline Steps $\times 1000$, earlier that day & 0.01 & 0.01 & 0.49 \\
\hline Sleep duration, prior night & -0.01 & 0.02 & 0.76 \\
\hline Day in internship (linear) & 0.16 & 0.04 & $<.001$ \\
\hline \multicolumn{4}{|l|}{ Model fitting } \\
\hline ICC & 0.10 & & \\
\hline AIC & 7527.26 & & \\
\hline
\end{tabular}

$N$, number of subjects; obs, observations, which are smaller here than the total recorded due to lagging of predictor variables and listwise deletion on days during which data were missing; $b$, unstandardized coefficient; ICC, intraclass correlation coefficient; AIC Akaike information criterion

population is exacerbated by interns not adjusting or having difficulty adjusting their bedtime behaviors to accommodate work schedules enforcing earlier wake times. As the restriction of sleep leads to deterioration of neurocognitive functioning, ${ }^{27}$ trainees unable to adjust their sleep timing to their internship work schedules may be at risk for impaired neurocognitive function and medical errors. Moreover, we observed that drastic shifts in sleep timing characteristic of shift work led to reductions in sleep duration and mood. Importantly, these associations were found when intern sleep-wake timing was either earlier or later than pre-internship routine. However, stronger effects of misaligned sleep-wake timing on short sleep and negative mood were observed when intern sleep schedules were advanced rather than delayed, a finding that is especially concerning since intern midsleep was earlier than baseline patterns on $71.7 \%$ of nights during internship.

Evidence supporting the negative impact of circadian challenging work schedules on sleep and mood are consistent with data available from night and rotating shift workers. ${ }^{28,} 29$ Owing to high rates of rotating, night, and extended shift schedules, residents are at elevated risk for shift work disorder, a circadian rhythm sleep disorder. ${ }^{30,31}$ Given the effects of sleepiness and fatigue on physician performance,, , 9, 32 shift work disorder in this at-risk population may represent an under-recognized threat to resident health and patient welfare.

Physical activity during residency is an understudied area that warrants greater attention for its role in regulating intern stress and psychological wellbeing. Consistent with crosssectional research showing lower exercise rates for medical residents than medical students, ${ }^{6}$ our accelerometry-based estimates of daily steps demonstrated a $>10 \%$ decrease in average daily physical activity after transitioning to internship. As interns reported poorer mood when they were less active, this reduction in physical activity may increase risk for negative

\section{Bedtime and Waketime}

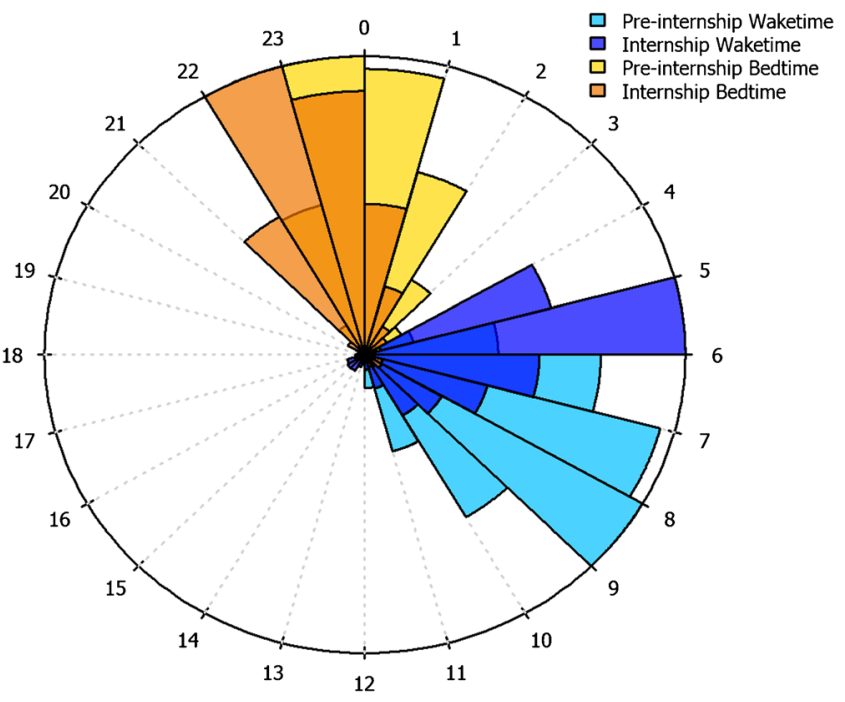

Midsleep

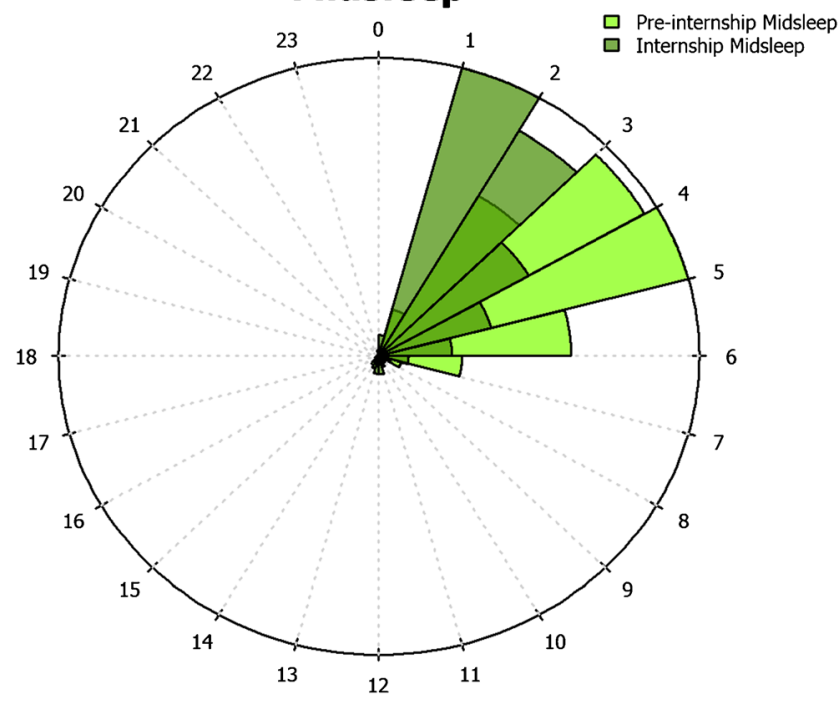

Figure 2 The distribution of bedtime, wake time, and midsleep before and during internship.

mood outcomes. These findings echo research highlighting the mood-improving effects of physical activity and exercise $^{33,}, 34$ and support recommendations for programmatic efforts in medical education to promote physical activity and increase access to exercise facilities to combat sedentariness and improve stress reduction. ${ }^{11}$

The present study should be interpreted in light of certain limitations. First, our sample comprised of 33 physicians in the internship program at the University of Michigan Medical School only; thus, rates of short sleep, physical activity, and other key factors should not be interpreted as prevalence rates for the overall intern population. While our large number of observations provides statistical power to assess withinsubjects effects, the limited number of subjects assessed limits the statistical power to detect between-subjects effects. 
Further, because the mixed models utilized just 33 observations for pre-internship factors (i.e., the number of subjects in the study), overfitting of between-subjects effects may have occurred, although the consistence of these findings with the extant literature may temper this concern. In addition, only data from pre-internship until December 31st were collected. Future investigations may benefit from continuous data collection throughout the entire intern year, which will provide insights into any potential changes in these relationships across the second half of internship. Further, it is unclear what, if any, effect self-monitoring may have had on interns' impressions of their mood, sleep, or physical activity and how this self-monitoring may have influenced intern behaviors. Though accelerometry-based sleep monitors exhibit good sensitivity for detecting sleep, they suffer from poor specificity and have been shown to overestimate sleep duration. ${ }^{15,16,35}$ Therefore, our data likely overestimate nightly sleep duration in this population. Future studies on nightly sleep in interns would benefit from sleep data collection using both subjective (e.g., sleep diaries) and objective (e.g., accelerometry or actigraphy) measures, as well as pre-internship assessment of circadian typology such as self-reported diurnal preference. Daily reports of shift schedules would improve future exploration of work hours and shift timing on intern health behaviors and outcomes. Lastly, although Mood 24/7 has shown promise in mobile mood monitoring, ${ }^{18}$ its use in research is limited and research on its clinical utility would benefit research using such prospective mobile data.

In summary, our findings suggest that interventions to increase sleep opportunity and physical activity may improve intern mood in this depression-vulnerable population. Physicians should be educated before internship on the importance of entraining their sleep-wake routines to earlier work schedules common to residency, and introduced to techniques to rapidly resynchronize their circadian rhythms when scheduled for early morning, night, or rotating shift work. ${ }^{36-38}$ Future research is needed to evaluate whether restructuring work schedules to best align with resident sleep-wake timing to permit interns to awaken close to their natural wake times, and designing shift changes to allow for adequate time for circadian resynchronization may improve both intern sleep and mood, and may protect against shift work disorder and reduce intern drowsiness.

\section{Acknowledgements:}

We would like to personally thank Zhuo Zhao, Elena Frank, Jad Elharake, and all of the research assistants who are instrumental to running the Intern Health Study.

Prior Presentations: These findings were presented at the 2016 National Network of Depression Centers annual conference in Boulder, CO.

Corresponding Author: Srijan Sen, MD PhD; Department of Psychiatry University of Michigan Medical School, Ann Arbor, MI, USA (e-mail: srijan@umich.edu).
Funders The funding sources had no role in the design and conduct of the study; collection, management, analysis, and interpretation of the data; preparation, review, or approval of the manuscript; and decision to submit the manuscript for publication. NIMH RO1 MH101459 (Sen), NHLBI T32 HL110952 (Pack), and the University of Michigan Taubman Institute Internal Grant (Sen).

Compliance with Ethical Standards: This study was approved by the University of Michigan IRB and all subjects provided informed consent after receiving complete description of the study.

Conflict of Interest: Dr. J Todd Arnedt has received research funding from Eisai Co., Ltd. All other authors report no conflicts of interest.

\section{REFERENCES}

1. Baglioni C, Battagliese G, Feige B, et al. Insomnia as a predictor of depression: a meta-analytic evaluation of longitudinal epidemiological studies. Journal of affective disorders. 2011;135(1):10-19.

2. Ströhle A. Physical activity, exercise, depression and anxiety disorders. Journal of neural transmission. 2009;116(6):777-784.

3. Sen S, Kranzler HR, Krystal JH, et al. A prospective cohort study investigating factors associated with depression during medical internship. Archives of general psychiatry. 2010;67(6):557-565.

4. Arora VM, Georgitis E, Woodruff JN, Humphrey HJ, Meltzer D. Improving sleep hygiene of medical interns: can the sleep, alertness, and fatigue education in residency program help? Archives of internal medicine. 2007;167(16):1738-1744.

5. Baldwin Jr D, Daugherty SR. Sleep deprivation and fatigue in residency training: results of a national survey of first-and second-year residents. Sleep. 2004;27(2):217-223.

6. Rye PL, Reeson ME, Pekrul CM, et al. Comparing health behaviours of internal medicine residents and medical students: an observational study. Clinical \& Investigative Medicine. 2012;35(1):40-44.

7. Mota MC, Waterhouse J, De-Souza DA, et al. Association between chronotype, food intake and physical activity in medical residents. Chronobiology International. 2016:1-10.

8. Olson EJ, Drage LA, Auger RR. Sleep deprivation, physician performance, and patient safety. CHEST Journal. 2009;136(5):1389-1396.

9. Weinger MB, Ancoli-Israel S. Sleep deprivation and clinical performance. Jama. 2002;287(8):955-957.

10. Kalmbach DA, Arnedt JT, Song PX, Guille C, Sen S. Sleep disturbance and short sleep as risk factors for depression and perceived medical errors in first-year residents. Sleep. 2017;40(3).

11. Williams AS, Williams CD, Cronk NJ, Kruse RL, Ringdahl EN, Koopman RJ. Understanding the exercise habits of residents and attending physicians: a mixed methodology study. Family medicine 2015;47(2): 118-123.

12. Hull SK, DiLalla LF, Dorsey JK. Prevalence of health-related behaviors among physicians and medical trainees. Academic Psychiatry 2008;32(1):31-38.

13. Sinsky C, Colligan L, Li L, et al. Allocation of physician time in ambulatory practice: a time and motion study in 4 specialties. Annals of Internal Medicine. 2016.

14. Guille C, Zhao Z, Krystal J, Nichols B, Brady K, Sen S. Web-based cognitive behavioral therapy intervention for the prevention of suicidal ideation in medical interns: a randomized clinical trial. JAMA Psychiatry. 2015;72(12):1192-1198.

15. Montgomery-Downs HE, Insana SP, Bond JA. Movement toward a novel activity monitoring device. Sleep and Breathing. 2012;16(3):913-917.

16. Ferguson T, Rowlands AV, Olds T, Maher $\mathbf{C}$. The validity of consumerlevel, activity monitors in healthy adults worn in free-living conditions: a cross-sectional study. Int J Behav Nutr Phys Act. 2015;12(1):42.

17. Adam Noah J, Spierer DK, Gu J, Bronner S. Comparison of steps and energy expenditure assessment in adults of Fitbit Tracker and Ultra to the Actical and indirect calorimetry. Journal of medical engineering \& technology. 2013;37(7):456-462.

18. Foreman AC, Hall C, Bone $\mathbf{K}$, Cheng J, Kaplin A. Just text me: using SMS technology for collaborative patient mood charting. Journal of Participatory Medicine. 2011;3:e45.

19. Walch OJ, Cochran A, Forger DB. A global quantification of "normal" sleep schedules using smartphone data. Science Advances. 2016;2(5):e1501705. 
20. Team RC. R: a Language and Environment for Statistical Computing. R Foundation for Statistical Computing, Vienna, Austria. 2013. ISBN 3900051-07-0; 2014.

21. Krueger C, Tian L. A comparison of the general linear mixed model and repeated measures ANOVA using a dataset with multiple missing data points. Biological research for nursing. 2004;6(2): 151-157

22. Singer JD, Willett JB. Applied Longitudinal Data Analysis: Modelling Change and Event Occurrence. New York: Oxford University; 2003.

23. Sen S, Kranzler HR, Didwania AK, et al. Effects of the 2011 duty hour reforms on interns and their patients: a prospective longitudinal cohort study. JAMA internal medicine. 2013;173(8):657-662.

24. Rosen IM, Gimotty PA, Shea JA, Bellini LM. Evolution of sleep quantity, sleep deprivation, mood disturbances, empathy, and burnout among interns. Academic Medicine. 2006;81(1):82-85.

25. Kalmbach DA, Pillai V, Roth T, Drake CL. The interplay between daily affect and sleep: a 2-week study of young women. Journal of Sleep Research. 2014

26. Kalmbach DA, Arnedt JT, Swanson LM, Rapier JL, Ciesla JA Reciprocal dynamics between self-rated sleep and symptoms of depression and anxiety in young adult women: a 14-day diary study. Sleep Medicine 2017;33:6-12.

27. Van Dongen HP, Maislin G, Mullington JM, Dinges DF. The cumulative cost of additional wakefulness: dose-response effects on neurobehavioral functions and sleep physiology from chronic sleep restriction and total sleep deprivation. SLEEP 2003;26(2):117-129.

28. Drake CL, Roehrs T, Richardson G, Walsh JK, Roth T. Shift work sleep disorder: prevalence and consequences beyond that of symptomatic day workers. SLEEP. 2004;27(8):1453-1462.
29. Kalmbach DA, Pillai V, Chen P, Arnedt JT, Drake C. Shift work disorder, depression, and anxiety in the transition to rotating shifts: the role of sleep reactivity. Sleep Medicine. 2015;16(12):1532-1538.

30. Association AP. DSM 5. American Psychiatric Association; 2013.

31. Medicine AAoS. International Classification of Sleep Disorders-Third Edition (ICSD-3). AASM Resource Library [online]. 2014.

32. Lockley SW, Barger LK, Ayas NT, Rothschild JM, Czeisler CA, Landrigan CP. Effects of health care provider work hours and sleep deprivation on safety and performance. Joint Commission Journal on Quality and Patient Safety. 2007;33(Supplement 1):7-18.

33. Giacobbi PR, Hausenblas HA, Frye N. A naturalistic assessment of the relationship between personality, daily life events, leisure-time exercise, and mood. Psychology of Sport and Exercise. 2005;6(1):67-81.

34. Peluso MAM, Andrade LHSGd. Physical activity and mental health: the association between exercise and mood. Clinics. 2005;60(1):61-70.

35. Meltzer LJ, Hiruma LS, Avis K, Montgomery-Downs H, Valentin J. Comparison of a commercial accelerometer with polysomnography and actigraphy in children and adolescents. Sleep. 2015;38(8): 1323.

36. Serkh K, Forger DB. Optimal schedules of light exposure for rapidly correcting circadian misalignment. PLoS Comput Biol. 2014;10(4):e1003523.

37. Eastman CI, Burgess HJ. How to travel the world without jet lag. Sleep medicine clinics. 2009;4(2):241-255.

38. Crowley SJ, Lee C, Tseng CY, Fogg LF, Eastman CI. Combinations of bright light, scheduled dark, sunglasses, and melatonin to facilitate circadian entrainment to night shift work. Journal of Biological Rhythms. 2003; 18(6):513-523. 